\title{
A large crater as a probe of the internal structure of the E-type asteroid Steins
}

\author{
M. Jutzi ${ }^{1}$, P. Michel ${ }^{2}$, and W. Benz ${ }^{3}$ \\ 1 Earth and Planetary Sciences Department, University of California, 1156 High Street, Santa Cruz California 95060, USA \\ 2 University of Nice-Sophia Antipolis, CNRS UMR 6202 Cassiopée, Observatoire de la Côte d'Azur, BP 4229, \\ 06304 Nice Cedex 4, France \\ e-mail: michelp@oca.eu \\ 3 University of Bern, Physikalisches Institut, Sidlerstrasse 5, 3012 Bern, Switzerland
}

Received 26 October 2009 / Accepted 8 December 2009

ABSTRACT

\begin{abstract}
Context. The detection of a large crater ( $2 \mathrm{~km}$ in diameter) on Steins, a diamond-shape asteroid with a diameter of only about $4.6 \mathrm{~km}$, was a large surprise given the size of the asteroid itself. Steins belongs to the rare class of E-type asteroids, which had not been observed by any spacecraft before the Rosetta mission of the European Space Agency (ESA) in 2008.

Aims. We demonstrate that this large crater places constraints on both the internal structure of Steins and its age based on crater counting.

Methods. Numerical simulations of impacts were performed to reproduce the large crater, assuming four different initial internal structures of the asteroid: monolithic with or without microporosity, and rubble pile with or without microporosity.

Results. To reproduce this crater, Steins must be either a rubble pile, which also contains microporosity, or a monolithic body with or without microporosity. The lack of porosity in meteorite analogues favors a structure without microporosity, which, according to our results, must have been monolothic. Moreover, the asteroid would be transformed into a rubble pile structure as a result of the crater formation, allowing it to be reshaped in its current shape by the YORP spin-up thermal effect.

Conclusions. A combination of modeling and observations of surface features can thus serve as a probe of the internal structure of a small body and constrains its age estimate. Since the surface was totally refreshed when the large crater was formed, crater counting on Steins indicates the time that has passed since this impact event occurred.
\end{abstract}

Key words. methods: numerical - minor planets, asteroids - solar system: general - shock waves

\section{Introduction}

E-type asteroids are found mostly in the inner part of the main asteroid belt located between Mars and Jupiter. It is believed that they originate in the mantle of larger asteroids that were destroyed in the early history of the Solar System and are understood to consist mainly of silicate minerals with little or no iron content (Gaffey et al. 1992). At its closest approach, the space probe Rosetta flew by the E-type asteroid Steins at a relative speed of $8.6 \mathrm{~km} \mathrm{~s}$ and a distance of about $800 \mathrm{~km}$. The first images from Rosetta's OSIRIS imaging system and VIRTIS infrared spectrometer, derived from raw data on September 6th, 2008, provided spectacular results (Keller et al. 2009). In the images, several small craters on the asteroid were clearly visible in addition to one large crater $2 \mathrm{~km}$ in diameter very close to the rotational pole of the object. Compared to the asteroid size (of about $4.6 \mathrm{~km}$ ), this is very large and contrains the impact response of the body and its internal structure. Based on standard knowledge, this large crater is not expected to exist on a body so small. There is a size limit to the crater that a body can sustain, which depends on the impact response of the body and hence its internal structure (Michel et al. 2003).

Porous bodies have been proposed to survive high impact energy events and therefore host larger craters on their surfaces than non-porous ones (Housen \& Holsapple 2003). This idea is consistent with images from the NEAR probe (NASA) of the
$52 \mathrm{~km}$-size asteroid Mathilde, which detected five craters larger than $20 \mathrm{~km}$ on its surface. Because of the low estimated bulk density of this asteroid, a large fraction of its porosity was assumed to be present within (Yeomans et al. 1997). Impact experiments on a laboratory scale were then performed to characterize the impact response of porous material and showed that this material can undergo compaction caused by the crushing of pores during impacts, which can explain that they survive larger craterforming events (Housen \& Holsapple 2003). However, extrapolating laboratory-scale experiments to large-scale events such as those on a kilometre-size asteroid is subject to large uncertainties.

We included a model of fragmentation of porous materials (Jutzi et al. 2008) in our 3D numerical hydrodynamical code based on the Smooth Particle Hydrodynamics (SPH) technique (Benz 1990; Monaghan 1992), which already contained a model of fragmentation of non-porous materials (Benz \& Asphaug 1994). This new model was then tested in the laboratory by comparing with impact experiments on pumice targets (Jutzi et al. 2009). In this case, porosity is defined as the presence of pores whose size is smaller than both the thickness of the shock front and the numerical resolution. As demonstrated by experiments, these pores may be the origin of energy dissipation caused by compaction during impacts. We use our newly developed code to study whether this porosity can explain the large craters observed on some asteroids. Because little is known 
Table 1. Internal structures and material properties used in the simulations.

\begin{tabular}{cccc}
\hline \hline Simulation & Structure & Microporosity & Strength \\
\hline 1 & Monolithic & $25 \%$ & low \\
2 & Monolithic & $25 \%$ & high \\
3 & Monolithic & $0 \%$ & high \\
4 & Rubble-pile & $25 \%$ & low \\
5 & Rubble-pile & $25 \%$ & high \\
6 & Rubble-pile & $0 \%$ & high \\
\hline
\end{tabular}

Targets with a rubble-pile structure have a fraction of macroscopic voids of $15 \%$. See Table 2 for definitions of low and high tensile strengths.

about the asteroid Steins, apart from its spectral properties and images of its surface, it is difficult to estimate its internal structure and potential porosity. Information about its bulk density at least would be required to infer its porosity by comparing with the density of meteorite analogues. However, since the asteroid was too small, no results were obtained from the Rosetta's Radio Science Experiment and we are therefore left with the few but amazing images obtained during this fly-by. Fortunately, as we demonstrate in this paper, we can obtain some constraints on the true internal structure of Steins from the presence of a large crater and our ability to estimate the impact response of a small body based on assuming different kinds of internal structure. The spectral type of Steins is among the least understood so far in terms of physical properties.

\section{Models of Steins' internal structure}

As a representation of Steins, we used an ellipsoidal shape whose dimensions had already been estimated by ground-based observations (Lamy et al. 2008). Although Steins has a diamondlike shape, we believe that this difference does not play a significant role in the problem we investigate, i.e., the high size ratio of the crater to the whole body. Moreover, the present shape of Steins resembles that produced by spin-up due to the thermal YORP (Yarkovsky-O'Keefe-Radzievskii-Paddack) effect on gravitational aggregates or rubble piles (Walsh et al. 2008), and this spin-up reshaping may well have occurred after the crater was formed. An additional advantage of this scenario is that a large crater can modify the value of the inertia tensor and on a long term, while spinning up, the rotation will adhere to the mode of rotation about the shortest axis (called short-axis mode, or SAM) of the inertia tensor (Vokrouhlicky, private communication). Therefore, a crater formed at any given latitude on the surface is likely to become centered on this rotation axis, as observed for Steins, and to conserve its original shape because of its large size. This would also explain the otherwise very unlikely event of an impact occurring almost exactly along the rotation pole of the body given the average impact geometries expected between asteroids (Bottke et al. 1994).

We searched for internal structures and impact conditions that allow us to form the large crater observed on Steins. The different internal structures used to perform the 3D SPH simulations are indicated in Table 1. Both monolithic and rubblepile (macroporous) structures were considered, either with or without microporosity (see Fig. 1). The material parameters are those that allowed us to verify our numerical code by confrontation with laboratory experiments, i.e., basalt for nonmicroporous bodies and pumice for microporous ones. The combination of either non-microporous, microporous and/or rubble-pile like structures in addition to low and high strength
Table 2. Definitions of low and high tensile strengths. (See text for details).

\begin{tabular}{cccc}
\hline \hline Tensile strength & $m$ & $\begin{array}{c}k \\
\left(\mathrm{~cm}^{3}\right)\end{array}$ & $\begin{array}{c}\sigma_{T}(3 \mathrm{~cm}) \\
\left(\mathrm{dynes} / \mathrm{cm}^{2}\right)\end{array}$ \\
\hline low & 9.5 & $8 \times 10^{37}$ & $\approx 3.5 \times 10^{7}$ \\
high & 9.5 & $3 \times 10^{28}$ & $\approx 3.5 \times 10^{8}$ \\
\hline
\end{tabular}
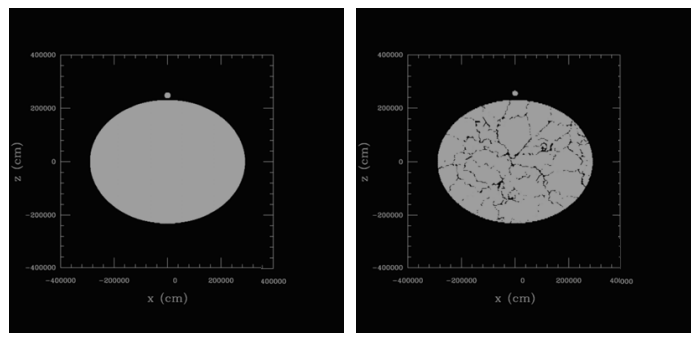

Fig. 1. 2D slices of the 3D Steins models showing their initial shape and structure. The internal structure is ether monolithic (left) or rubble pile (right) as defined in Table 1.

microporous material leads to six different targets (note that in the following, the term porous will be related to microporosity, unless stated otherwise). The rubble-pile structure is introduced by generating pre-shattered targets (as introduced by Michel et al. (2003)) and by removing the damaged particles between the intact blocks, producing large voids (we assume a $15 \%$ void fraction). The amount of microporosity, when present, was fixed at $25 \%$. While dark type asteroids are often assumed to have a high fraction of porosity $(>40 \%)$ based on measured bulk densities and comparison with carbonaceous chondrites (their meteorite anologues; Barucci et al. (2008)), bright type asteroids are expected to contain a smaller fraction of porosity (in particular microporosity), and this is particularly the case of E-type asteroids, even if no bulk density has yet been measured for any of them. However these bodies are generally supposed to be evolved, and their meteorite analogues to be enstatite achondrites (Britt \& Consolmagno 2000), which do not consist of porous material. Therefore, while searching for internal structures that would permit the formation of a large crater, we tried to remain as close as possible to the common belief that E-type bodies do not contain a large fraction of microporosity. If we find that the amount of porosity we consider is enough to produce the observed large crater, then increasing the porosity would make it easier, and therefore searching for the minimum amount required seemed a reasonable strategy. Regarding the assumed tensile strength, which is also unknown, we assumed both a low and a high value, as defined in Table 2. According to our fracture model, the tensile strength of a body depends on the parameters of the initial flaw distribution and is also volume dependent. This distribution, called a Weibull distribution, implies that the average tensile strength is given by $\sigma \approx(k V)^{-1 / m}$, where $V$ is the volume of the body, and $m$ and $k$ are the Weibull parameters. As a reference, we indicate the approximate tensile strength of a 3-cm target, $\sigma_{T}(3 \mathrm{~cm})$.

Figure 1 shows the two kinds of bodies that were considered. The number of SPH particles used is $5 \times 10^{6}$ for the monolithic targets and $4.25 \times 10^{6}$ for the rubble-pile ones. Their bulk density is assumed to be $2 \mathrm{~g} / \mathrm{cm}^{3}$. 

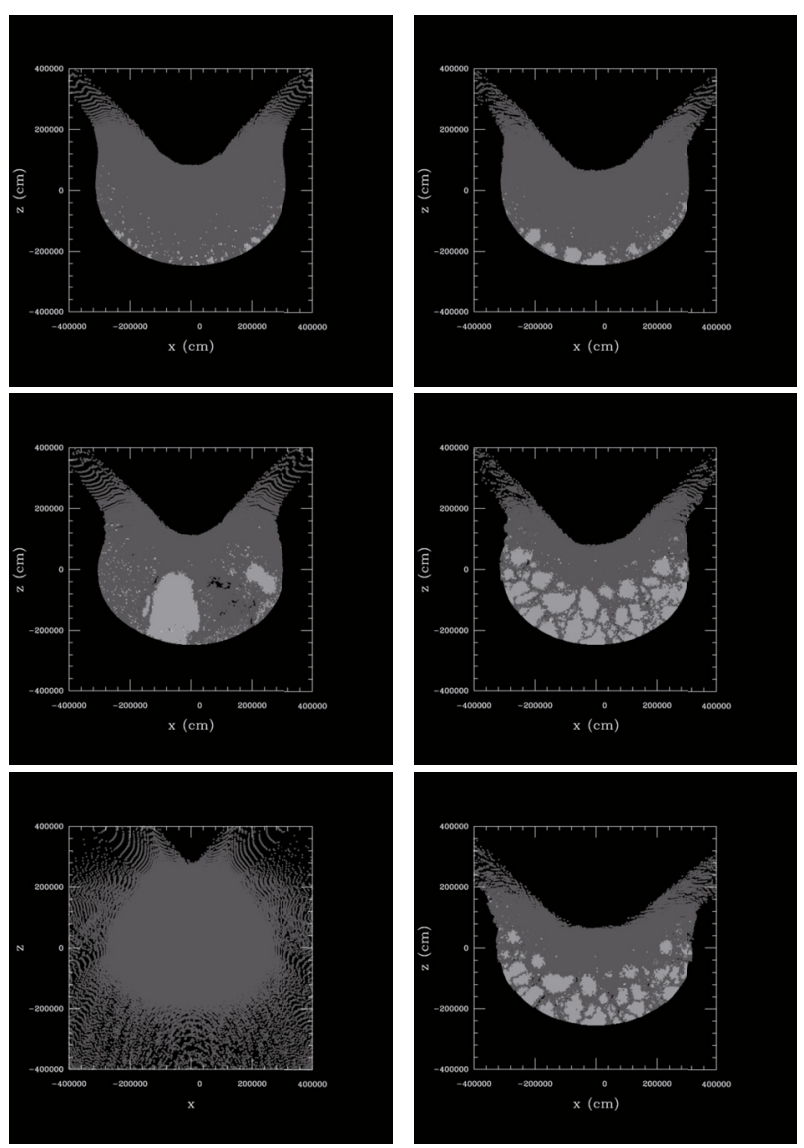

Fig. 2. Degree of damage (in gray scale) at $200 \mathrm{~s}$ from the time of impact. Dark-gray areas are damaged, while light-gray ones remain undamaged. The internal structure is monolithic (left) or rubble-pile (right). Top: microporous (25\%), low strength; middle: microporous (25\%), high strength; bottom: non-porous, high strength (see Table 2 for definitions of low and high strengths).

\section{Results}

All simulations started with a 180 meter-size non-porous (basalt) spherical projectile impacting head on at a speed of $5 \mathrm{~km} \mathrm{~s}^{-1}$, which corresponds to the average impact speed between asteroids in the main belt (Bottke et al. 1994). The simulations were stopped after $200 \mathrm{~s}$ from the time of impact in all cases considered. Consequently, the shape of the crater (and the entire body) does not necessarily correspond to the final shape. After $200 \mathrm{~s}$, fragments that do not escape may still have significant velocities. Hence, these fragments will move some distance before being reaccumulated by the body. This could potentially lead to a significant late-time change of shape. However, by looking at the shape of the body using velocity cut-offs significantly lower than the escape velocity, we concluded that reaccumulation is unlikely to alter dramatically the overall shape of the body.

Figure 2 shows the amount of damage and shape of the body at the end of the simulations. In Fig. 3, the fraction of escaping particles is indicated, and Fig. 4 shows a three-dimensional view of the targets where the escaping particles have been removed. We find that the bodies containing microporosity (monolithic or rubble pile) are able to survive this event and allow the formation of a crater with a size comparable to the observed one. Then, perhaps in contrast to expectations, we also found that this large crater can be formed using a non-porous monolithic body, while we found no solution with a non-porous rubble-pile. We also considered an impact angle of 45 degrees and a monolithic,
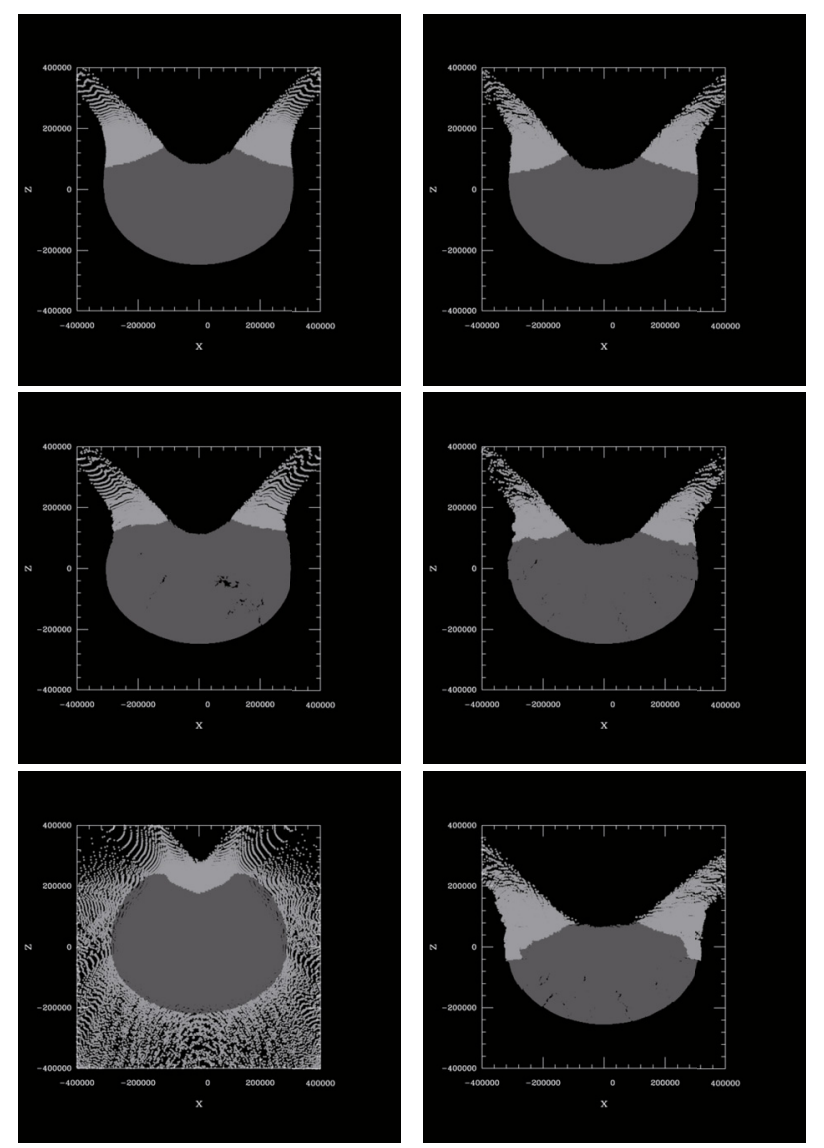

Fig. 3. Same as Fig. 2 but the escaping particles (light-gray) and bound ones (dark-gray) are shown. To compute the fraction of the material that escapes, the iterative method based on energy balance introduced by Benz \& Asphaug (1999) was used.

microporous structure. We found that this oblique impact leads to a slightly asymmetric crater but that otherwise the outcome is comparable to that of a head-on impact (in the target with the same material properties).

Thus, according to these simulations, which deserve a more extensive investigation over a much larger parameter space, it is not as difficult as one expects to produce a crater of this size on such a small body. However, there are wide variations in both the formation mechanism of the crater and the remaining body's properties for different assumed internal structures and strengths of the material. In the targets containing microporosity, the crater is formed by compaction of the porous material. For a non-porous monolithic body, the particular form of the velocity field generated by the impact leads to a crater-like feature (after removal of the escaping material). In this case, the crater is therefore solely formed by ejection of material.

In all cases considered, the final body is damaged considerably by the impact and will probably become a rubble-pile. We also find that the monolithic body conserves its original shape, apart from the new crater, and loses surface particles everywhere, like an onion after its first layer is removed. This is because the shock propagates coherently throughout the whole body therefore affecting it all. After the impact, the entire surface of the body would then be covered with freshly exposed material and appear spectrally homogeneous. This appears to be consistent with spectral observations performed by the OSIRIS camera on Rosetta, which are indicative of spectral homogeneity across Steins' surface (Kueppers et al. 2009). However, for 

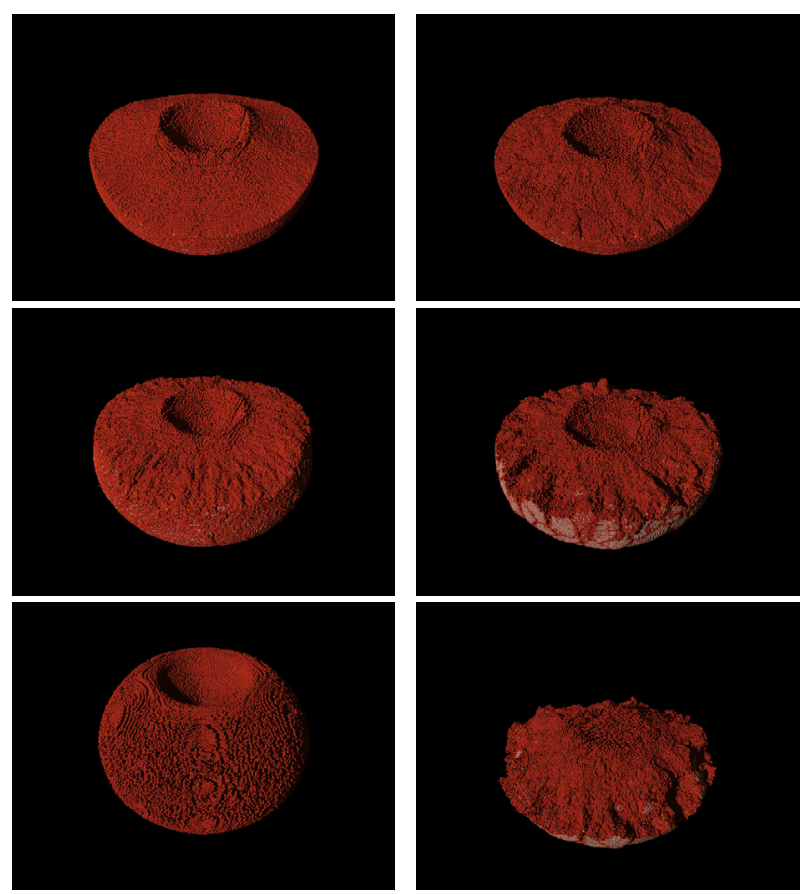

Fig. 4. 3D views of the targets after 200 s. The escaping particles (lightgray particles of Fig. 3) were removed. Red colour indicates fully damaged material, while white represents intact material. Internal structures and material properties are the same as in Figs. 2 and 3. As a result of the impact, all targets are at least partially damaged and originally monolithic ones are transformed into rubble-piles.

E-type materials, space weathering is poorly understood and it is unclear whether it would significantly affect the spectral properties of the exposed material as it does for other taxonomic types, such as S-types (Vernazza et al. 2009). Therefore, one cannot firmly conclude, based on this homogeneity, that the whole surface has been space weathered for the same amount of time, which would be the case in a scenario that starts from a nonporous monolithic structure. In contrast, in a porous/rubble pile body, ejection occurs only on the sides of the crater because of higher shock attenuation, which produces a different final shape, and also, old surface materials that survive. Even if the observed homogeneity is not used as a constraint, since after all meteorite analogues of E-class asteroids suggest that this class does not contain microporous bodies, we conclude that its original structure is most likely to have been a nonporous monolithic before Steins' large crater formed. In all cases, Steins' shape can be explained by YORP spin-up. The original monolithic non-porous structure of Steins is fully damaged by the impact that formed the crater (see Fig. 2). As a result, the asteroid is transformed into a rubble pile, which is necessary for YORP spin-up to produce the current shape (Walsh et al. 2008).

\section{Implications}

These results have several implications. The average collisional lifetime of a main belt asteroid of Steins' size is about 2.2. Gyr (Marchi et al. 2006), and therefore one may conclude that Steins is a fragment of a larger catastrophically disrupted body. Numerical simulations of catastrophic disruptions designed to reproduce $\mathrm{S}$-class asteroid family properties indicate that most fragments larger than a few kilometres in size are produced by the reaccumulation of smaller ones, and are consequently rubble piles (Benz \& Asphaug 1999; Michel et al. 2001). Our results suggest that Steins may have been monolithic when the impact at the origin of the crater occurred and thus indicate that E-type differentiated parent bodies may break up to allow intact fragments as large as Steins to be generated. The second implication is that the event that formed the crater produced a great amount of damage within the asteroid and it is probable that all craters present on the surface would have been erased by this event, either directly or because of strong seismic shaking. The craters observed in the images provided by Rosetta must therefore have been produced after. This has significant implications for Steins' age estimate. Our results imply that on the sole basis of craters, the estimated age will correspond only to the date at which the impact event that generated the large crater took place. This is an interesting result because if YORP spin-up produced the final shape of Steins after the impact event, as our results suggest, then one can estimate the YORP efficiency (timescale) that would allow Steins to assume this shape.

\section{Conclusion}

Numerical simulations can thus be used as probes of the internal structure of small bodies when only images are available, although additional measurements such as high precision spectral data, bulk density by means of radio science experiments, and/or comparison with meteorite analogues can allow a more robust discrimination between likely models. We can then hope that future space missions will allow us to continue improving our characterization of the internal properties of asteroids, especially those whose spectral type is poorly understood.

Acknowledgements. P.M. acknowledges the French Programme National de Planétologie and the cooperation program CNRS-JSPS 2008-2009. M.J. and W.B. acknowledge support from the Swiss National Science Foundation.

\section{References}

Barucci, M. A., Fornasier, S., Dotto, E., et al. 2008, A\&A, 477, 665 Benz, W. 1990, in Numerical Modelling of Nonlinear Stellar Pulsations Problems and Prospects, ed. J. R. Buchler, 269

Benz, W., \& Asphaug, E. 1994, Icarus, 107, 98

Benz, W., \& Asphaug, E. 1999, Icarus, 142, 5

Bottke, W. F., Nolan, M. C., Greenberg, R., et al. 1994, Icarus, 107, 255

Britt, D. T., \& Consolmagno, G. J. 2000, Icarus, 146, 213

Gaffey, M. J., Reed, K. L., \& Kelley, M. S. 1992, Icarus, 100, 95

Housen, K. R., \& Holsapple, K. A. 2003, Icarus, 163, 102

Jutzi, M., Benz, W., \& Michel, P. 2008, Icarus, 198, 242

Jutzi, M., Michel, P., Hiraoka, K., Nakamura, A. M., \& Benz, W. 2009, Icarus, 201, 802

Keller, H. U., Coradini, A., Barbieri, C., et al. 2009, in BAAS, 41, 564

Kueppers, M., Fornasier, S., Schroder, S., et al. 2009, in AAS/Division for Planetary Sciences Meeting Abstracts, 1118

Lamy, P. L., Kaasalainen, M., Lowry, S., et al. 2008, A\&A, 487, 1179

Marchi, S., Paolicchi, P., Lazzarin, M., et al. 2006, AJ, 131, 1138

Michel, P., Benz, W., Tanga, P., et al. 2001, Science, 294, 1696

Michel, P., Benz, W., \& Richardson, D. C. 2003, Nature, 421, 608

Monaghan, J. J. 1992, ARA\&A, 30, 543

Vernazza, P., Brunetto, R., Binzel, R. P., et al. 2009, Icarus, 202, 477

Walsh, K. J., Richardson, D. C., \& Michel, P. 2008, Nature, 454, 188

Yeomans, D. K., Barriot, J., Dunham, D. W., et al. 1997, Science, 278, 2106 